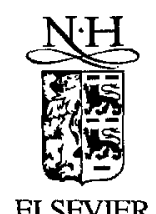

Physica D 89 (1996) 346-367

\title{
Spatio-angular order in populations of self-aligning objects: formation of oriented patches
}

\author{
Alex Mogilner, Leah Edelstein-Keshet \\ Department of Mathematics, University of British Columbia, Vancouver, BC V6T 1Z2, Canada
}

Received 18 June 1994; revised 26 April 1995; accepted 8 May 1995

Communicated by M. Mimura

\begin{abstract}
We consider a class of models for the dynamic behaviour of ensembles of objects whose interactions depend on angular orientations as well as spatial positions. The "objects" could be particles, molecules, cells or organisms. We show how processes such as mutual alignment, pattern formation, and aggregation are describable by sets of partial differential equations containing convolution terms. Kernels of these convolutions are functions that describe the intensity of interaction of the objects at various relative angles and distances to one another. Such models appear to contain a rich diversity of possible behaviour and dynamics, depending on details of the kernels involved. They are also of great generality, with applications in the natural sciences, including physics and biology. In the latter, the examples that fall into such class include molecular, cellular, as well as social phenomena. Analysis of the equations, and predictions in several test cases are presented. This paper is related to Mogilner and EdelsteinKeshet (1995) in which the spatially-homogeneous version of these models was investigated.
\end{abstract}

\section{Introduction}

The mathematical models dealt with in this paper describe a wide class of aggregation and alignment phenomena, motivated mainly by biological applications. We consider populations of interacting cells, organisms, or molecules in which individuals tend to aggregate and selfalign. Such phenomena are also well-known in physics (notably ferromagnets and liquid crystals) and hence we use the generic term objects in this paper when referring to the interacting particles, organisms, or cells. Our interest here is in the combined spatial and angular aspects of the order that emerges under different regimes when such objects interact. In all cases, we are inter- ested in self-organization of these populations. The order is not imposed by external bias or forces, but arises as a natural consequence of the interactions, starting from a chaotic or random state.

In the past, models for population distributions have tended to dwell exclusively on spatial distribution and temporal dynamics. However, there are instances in which the relative orientations of individuals have important influence on their dynamics and interactions. Typical examples are herds, fish schools, and other highly structured animal aggregates, where many members of the social group align with each other and move in a common direction (O'Brien, 1989; Katz et al., 1981). This type of behaviour allows 
the group to stay together, and it is often adopted as a defense against threats such as predation. (See, for example, Edmunds (1974) pp. 281, 282 for polarization in herds of wildebeest and zebras threatened by hyaenas.) Another example is the mutual contact and selfalignment in mammalian cells such as fibroblasts, which align with one another on a surface (Elsdale, 1972; see Fig. 1). The cells form dense patches within which they are more-or-less
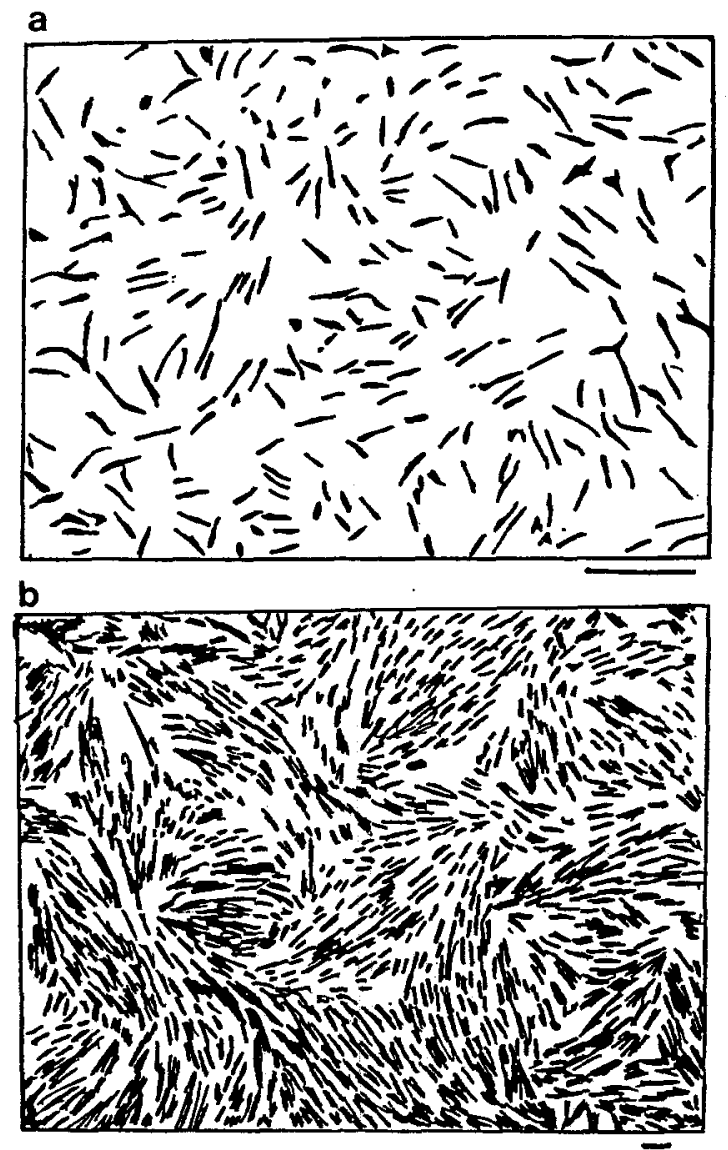

Fig. 1. Mammalian cells, known as fibroblasts demonstrate an alignment phenomenon similar to the one discussed in this paper. A population of cells that is initially oriented at random forms patches in which cells are lined up in parallel arrangements. (Note that this happens as the density of the cells increases.) The bar scales shown under the right hand corner of each box represent 1000 and $100 \mu$, respectively. (Sketched from a plate in Elsdale, 1973). aligned in a sea of rarefied (low density) randomly oriented cells. Because the type of interaction depends on relative orientations of contacting cells, we consider spatio-angular distributions of the cells. Empirical observations, or information about the phenomenon can be used to deduce the probability that a given contact will lead to a particular type of alignment event.

Models which describe interactions of individuals at various angles have been explored in a number of previous papers. Such modelling appeared in the context of branch interactions in a network of filaments in Edelstein-Keshet and Ermentrout (1989) and in fibroblast cultures in Edelstein-Keshet and Ermentrout (1990) (henceforth denoted $\operatorname{EKE}(90)$ ). A detailed analysis of the spatially homogeneous case was also given in Mogilner and Edelstein-Keshet (1995a).

The connection between interacting cells and models containing integral equations becomes apparent when one considers that a given cell can meet and interact with a neighboring cell of any other relative orientation. Each such event is associated with some probability that one of the two cells will turn and take on a new orientation. To account for all possible occurrences, one needs to sum up the probability of encounter, weighted by the likelihood of turning over all possible angles of contact. This reasoning leads to the formulation of integral equation models. Similar considerations apply in the binding of macromolecules which must take on the appropriate relative conformations to expose active sites to one another. The orientations of actin filaments which are bound and assembled into a scaffolding structure inside the cell by actin-binding proteins is one such example. This problem has been treated in detail in Civelekoglu and Edelstein-Keshet (1994).

The presence of an environmental gradient, or of some polarized background is not a requirement for the types of self-alignment discussed above, buit if it is present, it biases the selection 
of a direction with which to align. A discussion of self-alignment as a mechanism for enhancing chemotactic ability of social organisms such as schooling fish towards weak, noisy gradients is given in Grunbaum (1994). Locust swarms orient strongly to the direction of the wind. Flocks of migrating birds may use the earth's magnetic field as a directional cue. On the microscopic scale, motile mammalian cells (such as fibroblasts) tend to align strongly with grooves on an artificial substrate. All these cases are not directly addressed in this paper. We rather investigate the innate patterns of organization formed by a group of self-aligning individuals.

As will be discussed in a concluding section, models in this paper are also related to the physical kinetics of liquid crystals studied extensively for decades. (Greco and Marrucci, 1992; Lifshitz and Pitaevskii, 1981; Chandrasekhar et al., 1970; de Gennes 1974). Our approach does not, however, use the free energy functional, or the Lyapunov functional approach. We will discuss the two approaches at the conclusion of this paper. We rather use phenomenological model equations for the densities of objects which, in the physics literature, are often called rods, rodlike objects, rod-like molecules. The chief property of these objects is that they are elongated and have a natural axis of orientation. In the biological examples mentioned above one can distinguish an axis, as well as a front and a rear (e.g. "head" and "tail"). We will assume that the objects are axially symmetric (despite the fact that this is rarely the case in biological organisms which have clearly distinguishable dorsal, ventral characteristics.)

This paper is organized as follows: We introduce and describe the models in Section 2. In Section 3 we list some necessary mathematical facts. We perform linear stability analysis in Section 4. In Section 5 we speculate about conclusions of the analysis. After a brief discussion of cellular automata models in Section 6, we finish with a comparison of this approach with other physical and biological theories.

\section{Description of the models}

We represent populations of objects using a continuum description. Models we consider in the present paper share the following limitations: (1) At very low densities the stochastic processes of cell movement cannot be approximated by continuous PDEs. (2) At very high densities, topological packing constraints dominate and the models are no longer valid. (See Elsdale and Wasoff 1976, Onsager 1949). (3) The models do not distinguish between clusters of different sizes. This could be done by introducing the functions $P_{2}(\theta, t), P_{3}(\theta, t), P_{4}(\theta, t), \ldots$ to denote the density of clusters composed of two, three, four, . . , and $n$ objects at orientation $\theta$ but this would result in a system of infinitely many equations, a complication that we wish to avoid.

Density distributions that represent the population(s) of objects are functions of the space coordinate $r \in D$, orientational angle $\Omega \in S$ of the object's axis, and time $t$. The spatial domain, $D$ is either two dimensional (e.g. flat surface to which cells adhere in artificial in vitro growth conditions) or three dimensional (e.g. cells in vivo). In the two dimensional case, the angle describing the direction of an object is $\Omega \in$ $[-\pi, \pi]$ and we reserve the letter $\theta$ for it. In the three dimensional case, we have an angle in spherical coordinates, $\Omega \equiv(\phi, \theta)$ where $\phi \in$ $[0, \pi]$ is the colatitudinal angle, and $\theta \in[0,2 \pi]$ is the longitudinal angle. Since there is a one-toone correspondence between such directions and points on the unit sphere, the angular part of the distributions can be considered as functions on the unit sphere. We denote the angular space as $S$ in both two and three dimensions.

Throughout this paper we consider only the cases in which the range of effective interaction between the objects is at least a few orders of magnitude smaller than the size of the domain. This is a natural consequence of our assumption about the contact-like character of interaction between the objects. But note that by contactlike, we do not mean $\delta$-like interaction, as they 
are commonly called in physics, since in that case, integral terms disappear from the models. Rather we mean that the effective radius of interaction is of the same order as the length of the objects (usually much smaller than the size of the ensemble). This allows us to consider the spatial domain as infinite, and for the purposes of the linear stability analysis, to ignore boundary effects. The latter, however, cannot be ignored in the analysis of pattern formation, as will be briefly discussed later.

Three separate models are studied. In the first (Model I), we distinguish between two types of groupings characterized by densities of free versus bound objects. We can think of this subdivision as a phase separation: there is one dense phase, in which objects are "bound" and are consequently constrained in their motion, and a second, lower density phase in which the objects move independently. We use the notation $C(r, \Omega, t), P(r, \Omega, t)$ respectively for these density distributions. This model is related to the spatially homogeneous model described in EKE(90), Mogilner and Edelstein-Keshet (1995a).

In Model II and Model III we do not consider two separate phases, and in that case, we investigate a single density function, $C(r, \Omega, t)$. In Model II we consider the alignment process as a dynamic event which results from forces causing rotation of the interacting objects, rather than instantaneous "jumps" from one direction to another. Model III is a description of interactions of clusters of objects, in which the size of a cluster, as well as its position and orientation determines how it interacts with other oriented clusters.

\subsection{Model I: Instantaneous alignment}

The first model studied in this paper is an extension of the angular alignment model considered in the papers (EKE (1990), Mogilner and EK (1995a,b)). The variables in the model are:
Definitions:

$t=$ time,

$r=$ position,

$\Omega \equiv(\phi, \theta)=$ direction of orientation relative to some fixed coordinate axes,

$C(r, \Omega, t)=$ density of free objects at $r$ oriented with angle $\Omega$ at time $t$,

$P(r, \Omega, t)=$ density of bound objects at $r$ oriented with angle $\Omega$ at time $t$,

$K\left(r-r^{\prime}, \Omega, \Omega^{\prime}\right)=$ functional form of the rate that an object at $r^{\prime}, \Omega^{\prime}$ turns to $\Omega$ and moves to $r$ due to influence of object at $r, \Omega$,

$\beta=$ magnitude of the rate of alignment,

$\gamma=$ rate of exchange between bound and free cells,

$\mu_{1}=$ rate of random turning by free cells,

$\mu_{2}=$ rate of random walk (diffusion) of free cells.

The dynamic behaviour of the densities $C$ and $P$ is governed by the equations

\section{Model I}

$\left\{\begin{array}{c}\frac{\partial P}{\partial t}(r, \Omega, t)=\beta C K * C+\beta P K * C-\gamma P, \\ \frac{\partial C}{\partial t}(r, \Omega t)=\mu_{1} \Delta_{\Omega} C+\mu_{2} \Delta_{r} C-\beta C K * C \\ -\beta C K * P+\gamma P .\end{array}\right.$

In these equations, $\Delta_{\Omega}$ is the Laplacian in the angular variables, namely

$$
\begin{aligned}
& \Delta_{\Omega} \\
& = \begin{cases}\frac{\partial^{2}}{\partial \theta^{2}}, & \text { in 2D } \\
\frac{1}{\sin \phi} \frac{\partial}{\partial \phi}\left(\sin \phi \frac{\partial}{\partial f}\right)+\frac{1}{\sin ^{2} \phi} \frac{\partial^{2}}{\partial \theta^{2}}, & \text { in 3D. }\end{cases}
\end{aligned}
$$

The operator $\Delta_{r}$ is the Laplacian in spatial variables, i.e.,

$\Delta_{r}= \begin{cases}\frac{\partial^{2}}{\partial x^{2}}+\frac{\partial^{2}}{\partial y^{2}}, & \text { in 2D } \\ \frac{\partial^{2}}{\partial x^{2}}+\frac{\partial^{2}}{\partial y^{2}}+\frac{\partial^{2}}{\partial z^{2}}, & \text { in 3D. }\end{cases}$ 
$K *$ is a linear operator that depends on the orientation $\Omega, \Omega^{\prime}$ and on the distance $r-r^{\prime}$ between the interacting objects.

$$
\begin{aligned}
(K * f)(r, \Omega, t) \equiv & \int_{S} \mathrm{~d} \Omega^{\prime} \int_{D} \mathrm{~d} r^{\prime} K\left(r-r^{\prime}, \Omega, \Omega^{\prime}\right) \\
& \times f\left(r^{\prime}, \Omega^{\prime}, t\right) .
\end{aligned}
$$

Details about the kernel, $K\left(r-r^{\prime}, \Omega, \Omega^{\prime}\right)$ will be given below. The expression (2.4) is the influence of the distribution of material $f\left(r^{\prime}, \Omega^{\prime}, t\right)$ on the angle $\Omega$ at position $r$. Thus a term of the form $K * C$ represents the influence of the free object distribution at angle $\Omega$ and position $r$, and $\beta C K * C$ is the rate at which this influence results in free cells realigning and sticking to other cells at $\Omega, r$. (They then become bound cells.) A detailed derivation and description of this model with no space dependence appears in the previously cited papers.

The nonlinear terms in Eq. (2.1) are responsible for alignment of the objects. We showed in a previous publication (Mogilner and EdelsteinKeshet, 1995a,b) that the following situation occurs in the spatially homogeneous case $\left(\mu_{2}=\right.$ $0)$ : if $\mu_{1}$ is smaller than a critical value, the stability of the homogeneous state is broken, and pattern evolves in angular space. The alignment starts as a smooth bump in the distribution of the objects. As the governing parameter $\mu_{1}$ decreases, this bump turns into a narrow peak (or a number of peaks) so that the objects become fully aligned, and share a common axis (or common axes) of orientation.

We use the spatial Laplacian operator to capture a random motion of the object which does not include persistence in the direction of alignment, but rather pure random walk of the object's center of mass. In each experimental situation, there would be a specific type of stochastic process governing the motion of individual objects. Some stochastic processes would lead to Brownian motion, while others result in a persistent motion in random direction.
Further, random turning in general is described by more general operators, for example integral operators or their equivalents (see Murray, 1989, Section 9.5). Here we focus on the simplest situation, namely that of simple diffusion captured by the Laplacian operator. It will be seen below that there is a strong mathematical reason for choosing the Laplacian, namely that its eigenfunctions are identical to those of the integral operator of the model.

The form of the kernel $K\left(r-r^{\prime}, \Omega, \Omega^{\prime}\right)$ in our model is of crucial importance and deserves special explanation. The dependence on the radius vector, $r-r^{\prime}$ between the centers of mass of the objects, follows from the fact that the spatial region is assumed to be homogeneous with respect to its influence on the objects. If this homogeneity is broken (due to environmental bias or external force), then more general dependence $K\left(r, r^{\prime}, \Omega, \Omega^{\prime}\right)$ arises.

The convolution term describes an elementary process of alignment: two objects meet with initial directions $\Omega, \Omega^{\prime}$ and corresponding positions $r, r^{\prime}$. With probability $\alpha$, the objects continue moving with no interaction, and their directions are not changed. With equal probability $(1-\alpha) / 2$, the objects are attracted to either direction $\Omega$ and position $r$, or to direction $\Omega^{\prime}$ and position $r^{\prime}$. (The factor $(1-\alpha) / 2$ is absorbed into the constant $\beta$.)

Let us consider the situation when the distance between the objects is $L / 2$ (where $L$ is the length of the object), and the objects are parallel to each other. Then we distinguish between two possibilities: (1) the directions of the objects are normal to the radius vector between their centers-of-mass, or (2) they are parallel to the radius vector (see Fig. 2). The value of $K$ represents the probability amplitude of alignment. Experimental observations suggest that the greater the contact area between the objects, the greater is the probability of alignment. In the second situation, evidently this contact area is larger. For this reason, we can deduce that the 


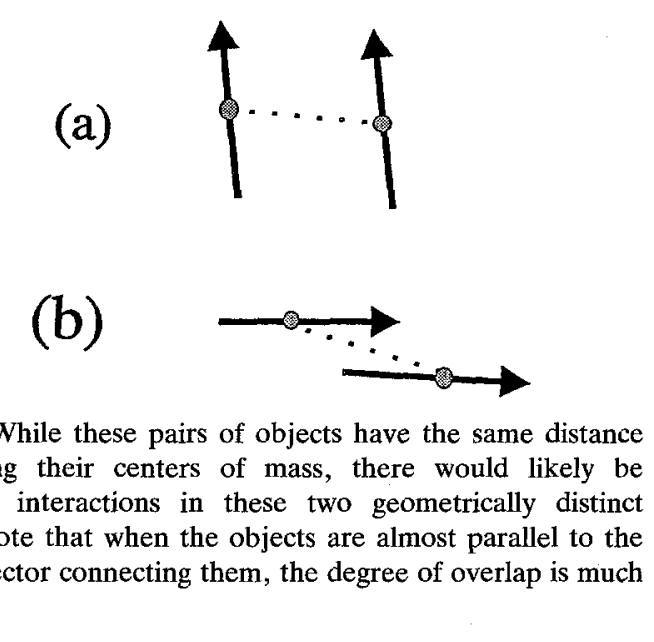

value of $K$ should be greater, though both values $\left(r-r^{\prime}\right)$ and $\left(\Omega-\Omega^{\prime}\right)$ are the same in the two cases. Thus it is reasonable to assume that the interaction depends on the radius vector, and two angles of the objects' axes relative to the radius vector connecting them.

For the sake of simplicity in this first investigation of the problem we will restrict attention to a somewhat less general form of the separable kernel,

$K\left(r-r^{\prime}, \Omega, \Omega^{\prime}\right)=K_{1}\left(r-r^{\prime}\right) K_{2}\left(\Omega-\Omega^{\prime}\right)$.

(The fully general situation will be discussed separately.) The strength of the interactions decreases as the distance between the objects grows. We assume that the effective range of interaction is of order $L$, where $L$ is the length of the object. For the spatial part, it is natural to assume the form

$K_{2}(r)=\exp \left(-r^{2} / 2\right)$,

where $r$ is measured in units of $L$. For the angular part of the kernel we use assumptions similar to (EKE90, Mogilner and EdelsteinKeshet 1995a). The behaviour of the models depends on the kernel $K_{1}$, which would be specific to the experimental situation. In the case of metazoan cell such as fibroblasts, alignment rates are lowest if the cells meet at $90^{\circ}$ (see Figs. 3a,b). In the case of actin fibers, crosslinking proteins allow fibers to interact and bind at different configurations, including parallel (bundling) and orthogonal (networking) structures. (Civelekoglu and EK, 1994). The kernel is different in that case (see Fig. 3c).

In parallel interactions, we must still consider a further distinction, namely whether alignment occurs only "head-to-head" or also "head-totail". The first case leads to a single humped kernel in the domain $-\pi<\theta<\pi$ (see Fig. 3a). The second case results in a double humped kernel (see Fig. 3b). These kinds of kernels lead to parallel alignment of cells. In the case of Actin, where interactions can occur between orthogonal fibers, two mutually orthogonal axes of orientation can be formed. The total mass of cells is equally distributed between these two axes. This case can be treated in the same way

(a)

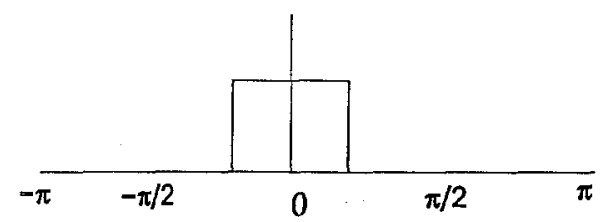

(b)

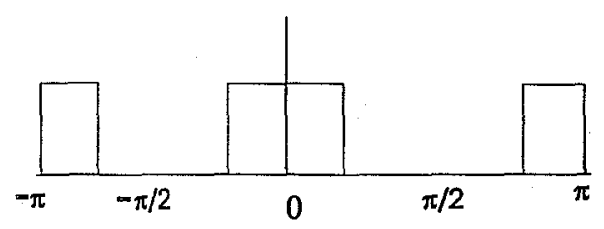

(c)

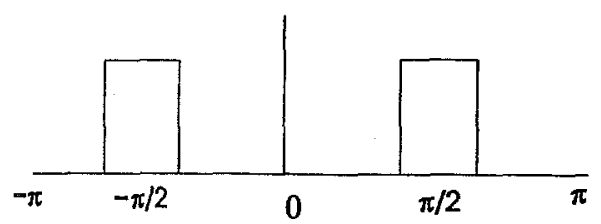

Fig. 3. The kernels $K$ used to represent the contact alignment phenomena are shown here as functions of the angle between cells. (In 2D the angle is $\theta$, and in 3D it is $\Omega$.) (a) Single humped kernel, representing alignment in which both cells are oriented head-to-head. (b) Double humped kernel which permits cells to align also in a head-to-tail configuration. (c) An orthogonal interaction kernel that plays a role in the model for actin alignment. 
(as shown in Civelekoglu and EK, 1994), by adjusting the kernel (see, for example Fig. 3c).

Let us introduce the mass of objects (at all angles) at location $r$ :

$M(r, t)=\int_{S}(C(r, \Omega, t)+P(r, \Omega, t)) \mathrm{d} \Omega$,

and the total mass of the whole ensemble,

$M=\int_{D} M(r, t) \mathrm{d} r$.

It is easy to check that the total mass is conserved in the model. In the usual biological situation, the objects proliferate (e.g. cells undergo cell-division) so that the total mass increases. (This mass growth slows down and stops when the density of objects reaches some critical level.) As the objects proliferate slowly, we assume that $M$ is the adiabatically varying variable. (I.e. for the analysis we take $M$ to be constant. Later, we consider what happens if $M$ is a slowly changing variable.)

For the purposes of analysis, it is convenient to recast the equations in a dimensionless form:

$$
\left\{\begin{aligned}
\frac{\partial P}{\partial t}(r, \Omega, t)=C K * C+P K * C-a P, \\
\frac{\partial C}{\partial t}(r, \Omega, t)=\epsilon_{1} \Delta_{\Omega} C+\epsilon_{2} \Delta_{r} C-C K * C \\
-C K * P+a P .
\end{aligned}\right.
$$

where $a=\gamma / \beta, \epsilon_{i}=\mu_{i} / \beta$.

Our description of the object ensemble implies that Model I has a non-local character. Indeed, the integral operator $K *$ defines the alignment as an instantaneous process rather than a local continuous one, having time dynamics. To put the elementary alignment process into the form of a dynamical and gradual change, we introduce a second model, below. At the same time, we no longer distinguish between free and bound objects, and consider only the density $C(r, \Omega, \theta)$ of one type of object. The definition and properties of the mass $M(r, t)$ are the same, but the terms with $P$ are omitted.

\subsection{Model II: Objects subject to alignment force}

This model is more physically realistic, since it takes into consideration real forces causing real velocities. We assume that the motion of the object consists of the following two parts: (a) rotation about the center of mass with "angular velocity" $\omega=\mathrm{d} \boldsymbol{\Omega} / \mathrm{d} t$; (b) drift of the center of mass with linear velocity $\boldsymbol{v}=\mathrm{d} \boldsymbol{r} / \mathrm{d} t$. (Here $\boldsymbol{r}, \boldsymbol{\Omega}$ are the spatial coordinate and direction of the object, respectively. In $2 \mathrm{D}, \omega=\mathrm{d} \theta / \mathrm{d} t$ is a scalar, and in $3 \mathrm{D}$ we define $\omega=\mathrm{d} \Omega / \mathrm{d} t$ as a tangent vector to the surface of the unit sphere, such that $\Omega$ is the angle between two points $\Omega_{1}, \Omega_{2}$ on the sphere. Then the direction of the vector $\boldsymbol{\Omega}$ is tangent to the arc joining $\Omega_{1}, \Omega_{2}$. Note that our definition of the angular velocity in $3 \mathrm{D}$ is different from the one common in mechanics).

As in Mogilner and Edelstein-Keshet (1995a), we will assume that velocities are proportional to corresponding forces:

$\omega \sim F_{\Omega}, \quad \boldsymbol{v} \sim F_{r}$.

The proportionality of the velocities to the driving forces stems from a number of biological cases such as molecular and cellular biology. Here very small objects move in highly viscous media, i.e. the motion is characterized by low Reynolds numbers and inertial forces can be neglected.

The forces $F_{\Omega}, F_{r}$ are assumed to be conservative. In that case, they derive from some underlying potential functions (as is true, for example for electrostatic forces). Thus we represent the forces as the gradients of these potentials (whose nature is not here characterised.)

$F_{\Omega}=\nabla_{\Omega} \tilde{W}, \quad F_{r}=\nabla_{r} \tilde{V}$.

Here, the angular gradient is given by

$\nabla_{\Omega}= \begin{cases}\frac{\partial}{\partial \theta} & \text { in } 2 \mathrm{D} \\ \hat{\phi} \frac{\partial}{\partial \phi}+\frac{1}{\sin \phi} \hat{\theta} \frac{\partial}{\partial \theta}, & \text { in } 3 \mathrm{D} .\end{cases}$ 
It is reasonable to assume that a given object at the angle $\Omega$, is subject to the effect of a potential that represents its cumulative interaction with the other objects. We assume linear superposition of forces holds. Then the potentials $\tilde{W}, \tilde{V}$ can be written in the form:

$\tilde{W}=W * C, \quad \tilde{V}=V * C$,

where $W * V *$ are the linear integral operators with the kernels $W_{1}(\Omega) W_{2}(r), V_{1}(\Omega) V_{2}(r)$, respectively, where

$V_{2}(r)=W_{2}(r)=\exp \left(-r^{2} / 2\right)$.

and $W_{1}(\Omega), V_{1}(\Omega)$ are the same as $K_{1}(\Omega)$. These kernels represent the potentials created by a single object at $\left(r^{\prime}, \Omega^{\prime}\right)$ acting on a single object at $(r, \Omega)$. The interaction between the objects is effective at distances of order $L$ between them and decreases quickly at large distances.

The motion induced by these forces sets up a drift of the objects both in physical space and in orientation space. Since the objects at a given position and angle all drift with the same mean rate, we can define fluxes (again in both angle, $J_{\Omega}$ and physical space, $J_{r}$ ) to describe the population drift.

$J_{\Omega}=C \omega, \quad J_{r}=C v$.

If we include both the divergence of the above convective fluxes and random motion, represented by the terms $\epsilon_{1} \Delta_{\Omega} C$ and $\epsilon_{2} \Delta_{r} C$ for the spatial and angular diffusion, in a balance equation for the distribution, $C(\Omega, r, t)$ we obtain the equation:

$\frac{\partial C}{\partial t}=\epsilon_{1} \Delta_{\Omega} C+\epsilon_{2} \Delta_{r} C-\nabla_{\Omega} \cdot J_{\Omega}-\nabla_{r} \cdot J_{r}$.

Using the equations $(2.10),(2.11),(2.13),(2.15)$ in the above leads, finally to

\section{Model II}

$$
\begin{aligned}
\frac{\partial C}{\partial t}= & \epsilon_{1} \Delta_{\Omega} C+\epsilon_{2} \Delta_{r} C-\nabla_{\Omega} \cdot\left(C \nabla_{\Omega}(W * C)\right) \\
& -\nabla_{r} \cdot\left(C \nabla_{r}(V * C)\right) .
\end{aligned}
$$

This equation describes the convectional drift of the objects in physical and angular space towards the points of the highest concentration, causing alignment and aggregation.

In the simplest 2D case, we can introduce $W^{\prime}$ and $V^{\prime}$ as follows:

$W^{\prime} \equiv \frac{\partial}{\partial \theta} W, \quad V^{\prime} \equiv \frac{\partial}{\partial r} V$.

Then these represent the forces corresponding to the above potentials. (Note that $W^{\prime}$ and $V^{\prime}$ are odd functions.) Then the nonlinear terms would have the forms

$$
\begin{aligned}
& \nabla_{\Omega} \cdot J_{\theta}=\frac{\partial}{\partial \theta}\left(C\left(W^{\prime} * C\right)\right), \\
& \nabla_{r} \cdot J_{r}=\frac{\partial}{\partial r}\left(C\left(V^{\prime} * C\right)\right) .
\end{aligned}
$$

The first term in (2.19) with a specific choice of kernel (independent of $r$ ), appeared in the models by (Grunbaum, 1994; Alt and Geigant, 1994) for chemotaxis and for Actin-myosin interactions, respectively.

\subsection{Model III: Interactions at discrete angles}

The third model is a simplification of the second model, in which objects jump instantaneously from their initial positions and orientations to ones acquired through the result of an interaction. We consider the interactions of clusters of objects, and assume that the size of the cluster influences its ability to attract objects. (Bigger clusters grow at the expense of smaller ones.) We use the same symbol, $C$ for density and consider only one type of object. The model again consists of a single equation. It turns out that if the nonlinearities are quadratic, and if no distinction is made between different types of objects, no pattern can form for the following reason: Suppose there are two clusters of fully aligned objects of different sizes. Because no turns to intermediate angles are allowed, the quadratic nonlinearities will not redistribute the objects, but diffusion will dissipate the peaks, so that pattern will not persist. By comparison, in models of (Geigant, Mogilner and Ladizhansky, 
1995) where turns to other angles are allowed, quadratic nonlinearities suffice to produce pattern. Here we consider another approach based on nonlinearities of higher order.

With the above considerations, we obtain the following equation:

\section{Model III}

$\frac{\partial C}{\partial t}=\epsilon_{1} \Delta_{\Omega} C+\epsilon_{2} \Delta_{r} C+C(Q(C) * C)$.

Here the integral term is defined as:

$$
\begin{aligned}
Q(C) * C= & \int_{S} \int_{D} L\left(C(r, \Omega)-C\left(r^{\prime}, \Omega^{\prime}\right)\right) \\
& \times G\left(r-r^{\prime}, \Omega-\Omega^{\prime}\right) \\
& \times C\left(r^{\prime}, \Omega^{\prime}, t\right) \mathrm{d} \Omega^{\prime} \mathrm{d} r .
\end{aligned}
$$

The kernel is now the product of two functions, one of which is responsible for the interaction dependence on the spatial and angular coordinates and has the same meaning and form as for the kernel $K$ above:

$G(\Omega, r)=G_{1}(\Omega) \mathrm{e}^{-r^{2} / 2}$.

The function, $L\left(C(r, \Omega)-C\left(r^{\prime}, \Omega^{\prime}\right)\right)$ reflects the tendency for the bigger cluster to grow at the expense of the smaller cluster.

This is a rough approximation of the process of fast motion of the small cluster of objects towards the more slowly moving big cluster, and their final merging. For this purpose, the function $L$ should be an odd one: $L(-C)=-L(C)$. At the same time, the symmetry of the function $L$ provides conservation of the total mass of the system. For the purposes of this paper, all we need to assume about the function $L$ aside from the fact that it is odd, monotonously increasing, and bounded. We do not need further specification of the function $L$.

In all three models, the nonlinear terms are favorable for the aggregation and alignment of the objects, while the diffusion terms cause dispersion of the objects, destroying order. We expect, then, that at large governing parameter values, $\epsilon_{1}, \epsilon_{2}$, the angularly and spatially homogeneous state is the only stable one. We will find that as the total mass in the system grows adiabatically, bifurcation occurs, the stability of the homogeneous state is broken, and a pattern emerges. In Section 4 we undertake linear perturbation analysis to investigate this phenomenon. Before doing so, however, we establish a number of results about the linearized problem that will be necessary for our analysis.

\section{Eigenfunctions of the operators}

We are considering an infinite spatial domain, so that the wavenumber $q$ is a continuous variable. Let us introduce the complete set of orthonormal functions:

$\left\{u_{q}(r) z_{n}(\Omega)\right\}, \quad q \in \mathbb{R}^{d}, n=0,1,2, \ldots$,

where

$u_{q}(r) \sim \exp \{\mathrm{i}(q \cdot r)\}$

and

$z_{n}=\left\{\begin{array}{ll}\mathrm{e}^{\mathrm{i} n \theta} & \text { in 2D, } \\ Y_{n}(\phi, \theta) & \text { in 3D, }\end{array} \quad n=0,1,2, \ldots\right.$

$Y_{n}$ are surface spherical harmonics (SSH), [Macrobert, 1967; Abramowitz and Stegun, 1964]. Our analysis is based on the following propositions:

Proposition 1. The set (3.1) is the set of eigenfunctions of the operator $\Delta_{r}$ with corresponding eigenvalues:

$\beta_{q}=-q^{2}$.

Proposition 2. The set (3.1) is the set of eigenfunctions of the operator $\Delta_{\Omega}$ with the corresponding eigenvalues:

$\alpha_{n}=\left\{\begin{array}{ll}-n^{2} & \text { in 2D }, \\ -n(n+1) & \text { in 3D },\end{array} \quad n=0,1,2, \ldots\right.$ 
Rigorous proof of the above two propositions can be found in any classical book on mathematical physics.

Proposition 3. The set (3.1) is the set of eigenfunctions of the operators $K *, W *, V *, G *$, with the corresponding eigenvalues: $\hat{K}(n, q), \hat{W}(n, q)$, $\hat{V}(n, q), \hat{G}(n, q)$ respectively. These eigenvalues are given by the following formula (in the case of $K$, for example):

$\hat{K}(n, q)=\hat{K}_{n} \mathrm{e}^{-q^{2} / 2}$.

Similar formulae hold for $\hat{W}, \hat{V}, \hat{G}$. The separability of the function $\hat{K}$ is a direct consequence of the separable nature of the kernel $K(\Omega, r)$. Here $\hat{K}_{n}$ is either the Fourier coefficient in the expansion of the kernel $K_{1}(\Omega)$ over cosines or sines or the coefficient in the Legendre expansion over the SSH (See Mogilner and Edelstein Keshet 1995a for details.) Eq. (3.6) follows from the fact that $\exp \left(-q^{2} / 2\right)$ is the Fourier transform of $\exp \left(-r^{2} / 2\right)$.

\section{Linear stability analysis}

In this section we consider only the $2 \mathrm{D}$ case. The 3D case is a clear generalization of our results. It is easy to see in all three models that the homogeneous solution (in both angle and space) is stationary. We are interested in the situation that pattern can arise. Our first step is to perform linear stability analysis to determine when the homogeneous solution becomes unstable.

Model I. We consider the weakly perturbed homogeneous pattern:

$\left[\begin{array}{l}P(r, \Omega, t) \\ C(r, \Omega, t)\end{array}\right]=\left[\begin{array}{l}\bar{P} \\ \bar{C}\end{array}\right]+\left[\begin{array}{l}P_{0} \\ C_{0}\end{array}\right] u_{q}(r) z_{n}(\Omega) \mathrm{e}^{\lambda t}$,

where $\bar{P}, \bar{C}$ obey the relations:
$M=\bar{P}+\bar{C}$,

$\bar{C}=\frac{a M}{a+M}$,

$\bar{P}=\frac{M^{2}}{a+M}$.

By substituting this into the system (2.9) and keeping terms linear in $P_{0}, C_{0}$ we obtain a condition under which $\lambda$ has a positive real part (implying growing perturbation, and thus instability). We introduce the function $\lambda \equiv \lambda_{n}(q)$ which we call the Linear Growth Rate (LGR). This function is obtained from the secular algebraic equation linking the values of $\lambda, n$ and $q$, and represents the rate of growth of pattern close to the homogeneous steady state. Note that conservation of mass in all three models implies that the homogeneous solution is neutrally stable to perturbation by the mode $n=0, q=0$ (that is, $\lambda_{0}(0)=0$ ).

The analysis is analogous to that in [EKE (1990)], and we get the instability criterion:

$\epsilon_{2} q^{2}<-\epsilon_{1} n^{2}+\hat{K}_{n} \mathrm{e}^{-q^{2} / 2}\left(1-\hat{K}_{n} \mathrm{e}^{-q^{2} / 2}\right)$.

Model II. Substituting the pattern

$C(r, \Omega, t)=\bar{C}+C_{0} u_{q}(r) z_{n}(\Omega) \mathrm{e}^{\lambda t}$,

into Eq. (2.17) and keeping the terms linear in $C_{0}$ we obtain the instability criterion:

$\epsilon_{2} q^{2}<-e_{1} n^{2}+\bar{C}\left(q^{2} \hat{V}_{n}+n^{2} \hat{W}_{n}\right) \mathrm{e}^{-q^{2 / 2}}$.

Model III. Substituting the pattern (4.4) into Eq. (2.20), using the fact that at small $C$ we have:

$L(C)=\eta C+O\left(C^{2}\right)$,

and keeping the terms linear in $C_{0}$, we get the instability criterion in the form:

$\epsilon_{2} q^{2}<-e_{1} n^{2}+\eta \bar{C}^{2}\left(1-\hat{G}_{n} \mathrm{e}^{-q^{2 / 2}}\right)$.

The criteria for all three models lead qualitatively to the same conclusions. The most convenient way to investigate the conclusions of these inequalities is by graphical methods. We introduce the complete bifurcation diagram (see Fig. 4). 


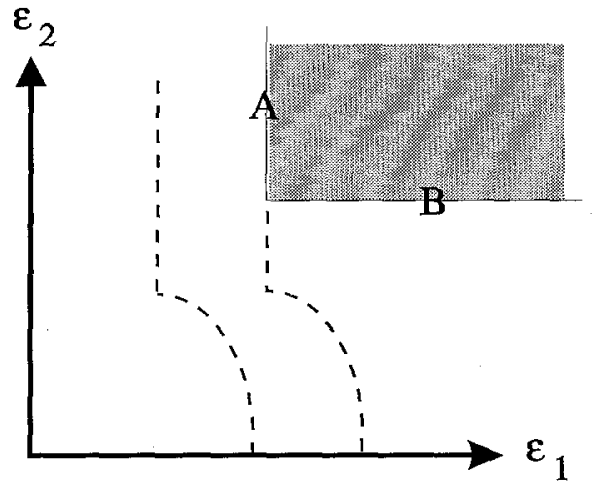

Fig. 4. A complete bifurcation diagram. A and B are the lines of primary bifurcations. Line $A$ corresponds to the growing mode $n \neq 0, q=0$. Line $\mathrm{B}$ corresponds to $n=0$, $q \approx 0$. The shaded region is the region of stability of the homogeneous state. The unshaded region corresponds to some non-homogeneous pattern. The dashed lines are secondary bifurcations. The vertical dashed lines correspond to $n \neq 0, q \neq 0$. The curved portion of the dashed lines corresponds to $n \neq 0, q \neq 0$.

This diagram represents the state of the system with all parameters fixed except two governing parameters, $\epsilon_{1}$ and $\epsilon_{2}$. The shaded area in the parameter space is the region of stability of the homogeneous solution. The border of this region is the set of primary bifurcation points. The unshaded region is the region of instability. The dashed lines are the set of secondary bifurcation points. As the total mass of the system slowly grows, and $\epsilon_{1}, \epsilon_{2}$ are decreasing, we cross either the line labelled (A) or the line labelled (B).

If we cross line A first then the mode corresponding to $n \neq 0, q=0$ breaks the stability. Then the angular pattern evolves while the spatial distribution remains homogeneous. I.e. the objects orient but they do not aggregate (see Fig. 5a). The nonlinear analysis of this situation is described in Mogilner and Edelstein-Keshet (1995a). We call this scenario $A$. If, on the other hand, we cross line $\mathrm{B}$, then the modes $n=0$, $q \approx 0$ (see Appendix) are responsible for the instabilities, then the angularly disordered pattern with the spatial inhomogeneities evolves. This means that the objects aggregate but they do not align. One would then see evolution of

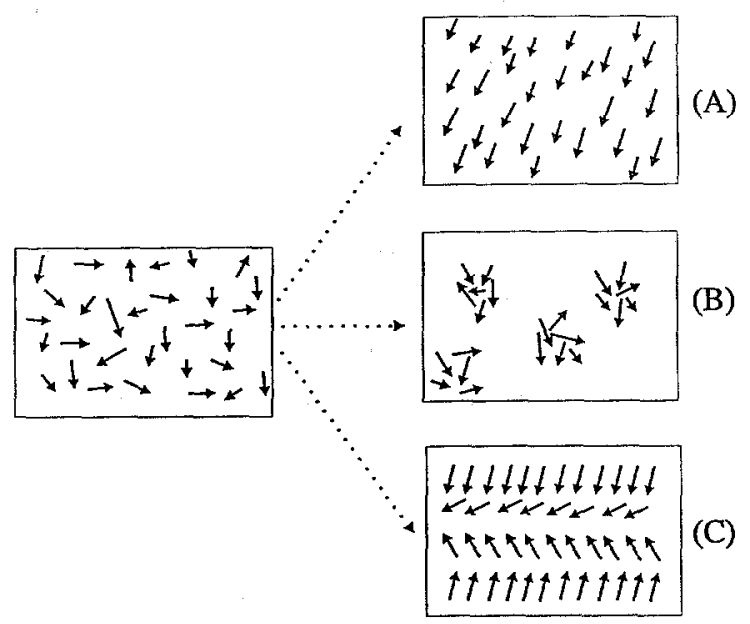

Fig. 5. Depending on the growth protocol, any one of three possible bifurcation scenarios can take place. Starting from an initially disordered state (left), bifurcation may lead to (A) formation of angular order in a distribution that is spatially homogeneous, (B) aggregation and formation of spatial inhomogeneities without angular order taking place, (C) formation of patches of aligned objects.

patches of objects within which the objects are not directionally ordered (see Fig. 5b). We call this scenario $B$. The detailed calculations leading to these conclusions are given in the Appendix.

We now briefly describe a more general situation. Let us consider the kernel $K(\Omega, r)$ such that its Fourier or Legendre coefficients have the form

$\hat{K}_{n} \exp \left(-\frac{1}{2} r_{n}^{2} q^{2}\right)$.

Here $r_{n}$ is the effective range of interaction (in units of $L$ ) within the angularly ordered phase. This means that spatio-angular dependence is more general and realistic than before. It can be shown that the kernel can be represented in the following form:

$K_{1}(\Omega) \exp \left(\frac{-r^{2}}{2 \kappa^{2}(\Omega)}\right)$,

where the effective radius of interaction, $\kappa$ depends in a nontrivial way on the angle. Thus we control not only the strength of the interaction, but also its effective range as a function of angle. We note that not all such kernels with 
the Fourier or Legendre coefficients given above by Eq. (4.8) are positive. Since kernels in our model are transition probabilities we must impose restrictions on acceptable values of $\hat{K}_{n}$ and $r_{n}$. It can be shown (we omit details of the calculations) that the complete bifurcation diagram has qualitatively the same form as the one in Fig. 4 but with displaced lines $A$ and B. In particular, the bigger is $r_{n}$, the larger the displacement from the origin of these lines. However, depending on $\hat{K}_{n}, r_{n}$, the diagram may change qualitatively in the vicinity of the intersection of lines A and B (see Fig. 6). The new primary bifurcation (line $C$ ) appears corresponding to the mode $n \neq 0, q \approx q^{(c)} \neq 0$. This line in general corresponds to the largest value of $r_{n}$.

If it happens that line $\mathrm{C}$ corresponds to the primary bifurcation or one of the secondary bifurcations, then the mode exp in $\theta \exp$ i $q r$ in $2 D$ and $Y_{n}(\Omega) \exp$ iqr in $3 \mathrm{D}$ is the leading one. Then spatio-angular order grows simultaneously. In this case, contrary to scenario B, the spatial density of the homogeneous state is not altered, but there exists long-range correlation between the axes of preferred orientation of the objects. In fact, the angle of preferred orientation changes periodically in space, with a characteristic period of order $L$, creating stripes (rolls) or spots (squares or hexagons). Schematically, the

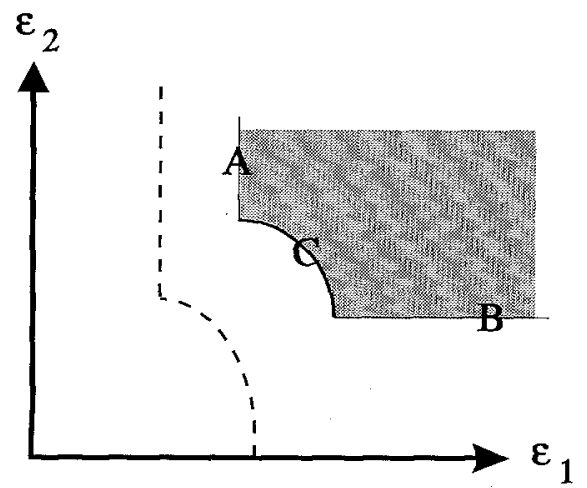

Fig. 6. The same as Fig. 4, but with an additional line for primary bifurcation (C). This line corresponds to the growing mode $n \neq 0, q \neq 0$. case of stripes is shown in Fig. 5c. This is strikingly similar to the experimental results of Tabony and Job (1990) in artificial polymerization of Tubulin. We call this scenario $C$.

Note that we do not consider structurally unstable situations when bifurcation is caused simultaneously by two different modes. This may happen, for example, if we cross at the point of intersection of lines $\mathrm{A}$ and $\mathrm{B}$. We also have to remember that linear stability analysis does not predict which pattern evolves in reality. It just gives us a strong hint about the form of the growing structure (see discussion for more details).

\section{Implications of the linear stability analysis}

Mathematical results obtained in Section 4 have the following qualitative interpretation: we have two diffusion coefficients, the rotational one $\mu_{1}$ with dimension $T^{-1}$ and the translational one, $\mu_{2}$ with dimension $l^{2} / T$. ( $l$ is the unit of length and $T$ is the unit of time.) We can then introduce an "intensity of interaction" of order $\beta M$ (which is the same for both spatial and angular interaction and has the dimension $T^{-1}$ ) and an "effective range of interaction" $L$. Three parameters with the dimension $T^{-1}$ define time dynamics in this system: $\mu_{1}, \mu_{2} / L^{2},(\beta M)$. (There will be an additional parameter, $\gamma$ in Model I. Then three parameters $\mu_{1} \gamma, \mu_{2} \gamma / L^{2}$, and $(\beta M)^{2}$ determine the dynamics of the system. If $\mu_{2} / L^{2}>\beta M$, over the effective range of the interaction translational diffusion destroys spatial order faster than the interaction between objects restores it. If simultaneously $\mu_{1}<\beta M$, the angular part of the interaction prevails over the rotational diffusion. In this situation scenario $A$ is realized, and the objects align first, before any spatial order is evident. Otherwise, if $\mu_{1}>$ $\beta M$ rotational diffusion destroys angular order, and $\mu_{2} / L^{2}<\beta M$ (spatial order is created), then we have scenario $B$, i.e. spatial aggregation is evident before any kind of angular order is seen. 
For example, the usual situation for molecules is $\mu_{1} L^{2} \sim \mu_{2}$ (see Landau and Lifshitz; (1956)). In this case the detailed structure of the kernel determines the bifurcation scenario. However, if for one reason or another in a biological system the parameters, $\mu_{1} / L^{2}$ and $\mu_{2}$ are very different, then clear predictions can be made without knowing the detailed form of the kernel. The specific Gaussian form of the kernels $K, W, V, G$ is not crucial for the results of this paper. The only important thing is the symmetry of the angular dependence, and the fact that the spatial dependence is a decreasing function of distance which falls to zero on a length scale of the order of $L$, the size of the object.

In a separate paper, we will investigate the character of the spatial bifurcation, and the spatial patterns evolved. We expect that this bifurcation will be a first order nonequilibrium phase transition. The smallness of the wavenumbers responsible for the bifurcation, implies a long length scale (relative to $L$ ) of the spatial pattern near criticality. This means that the length scale of the pattern may be comparable to the size of the spatial domain, and the effect of the boundaries could be significant. One of the implications is that irregular "droplets" containing large numbers of objects start to grow, and that a multitude of topological singularities can arise in the spatio-angular pattern (Cross and Hohenberg, 1993; Elsdale and Wasoff, 1976). This is similar to patterns formed in fibroblast cultures.

Characteristic sizes of patches of ordered objects that evolve and the time dynamics of this evolution are currently under investigation. It appears that the role of fluctuations is important near the nonequilibrium phase transition. It is also of interest to consider more realistic forms of the kernels $K\left(r-r^{\prime}, \Omega, \Omega^{\prime}\right)$. Throughout the present paper, we considered an isotropic domain. If there is anisotropy, the $O(n)$ symmetry is broken, and this must be reflected in the choice of kernel. We expect, further that nonlocal effects in the random motion of the objects will lead to nonlinear terms in the diffusion operators, which will cause important changes in the linear stability analysis and nonlinear bifurcation analysis. (See, for example Murray, 1989.)

In some systems in which there is substantial interest (for example aggregations of animals such as fish schools, bird flocks, or herds of mammals) free motion of the "objects" is not limited to diffusion, but contains an ordered component of persistent motion, with some intrinsic velocity. (See Alt and Pfistner, 1989). The cellular automata described below are an example of such systems. Including this motion in the models would lead to hydrodynamic-like equations and would reveal a number of new phenomena.

In all three models, we have at least two governing parameters proportional to the spatial and angular diffusion coefficients respectively. Due to the additional adiabatic growth of the total mass, the synchronous changes in these parameters causes one or another "growth protocol" (see Cross and Hohenberg, 1993) also called a "developmental pathway" (Segel, 1984) namely, a sequence of the spatio-angular bifurcations. The first bifurcation is one of three possible types described above. The final pattern, however, may be formed by secondary bifurcations. (Moreover, the linear instability at $q \approx 0$ may not lead to any stability break. In this case, the mode responsible for one of the secondary bifurcations will be responsible for the patterns evolved.) One of the possibilities is that if spatial patches of objects are formed in an angularly disordered state, then the conditions for alignment are most favorable within the patches, and the axes of orientation of objects in the different patches are not correlated. If, on the other hand, patches start to grow in a partially aligned ensemble of objects, then patches themselves have elongated forms and the axes of orientation of the objects within the patches are correlated between neighboring patches. (See Figs. 5c, 7.) This phenomenon is seen in cellular automata simulations. The full investigation of the final 
(a)

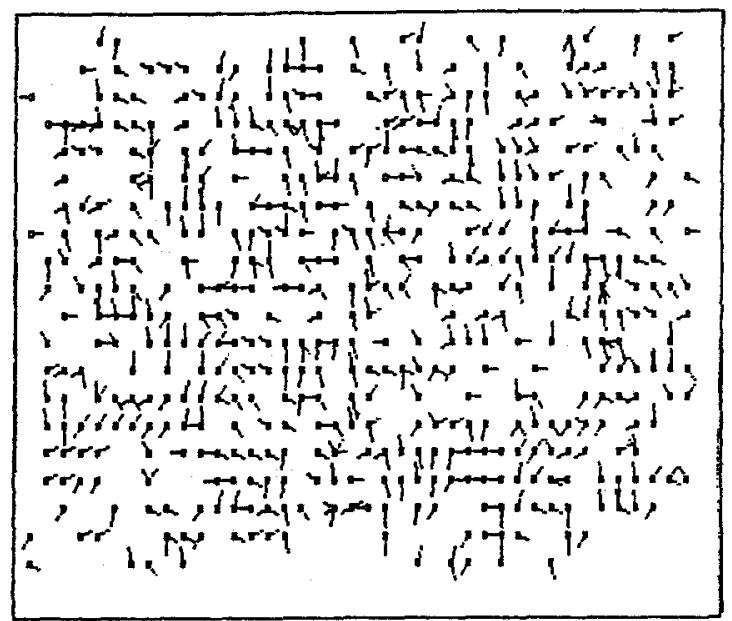

(b)

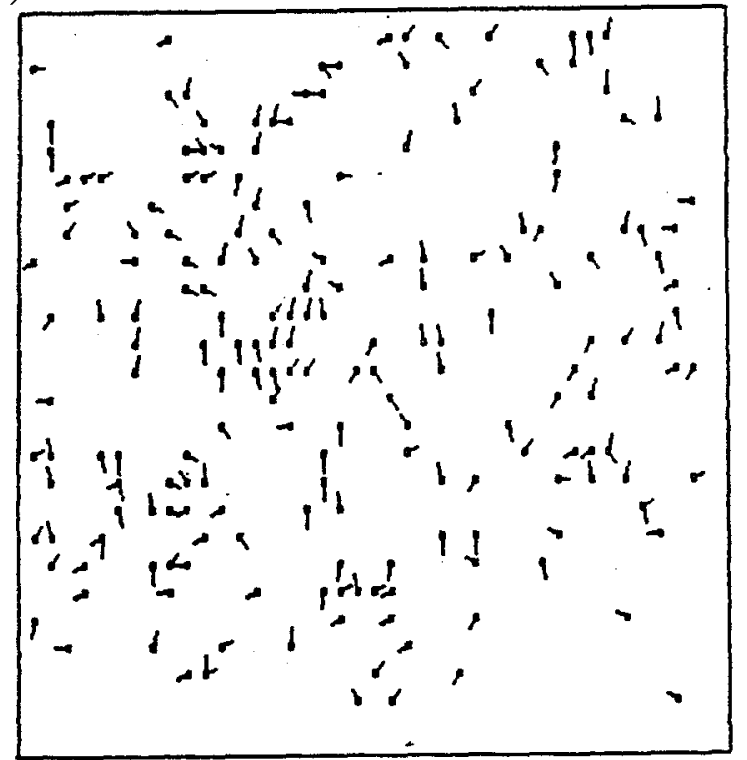

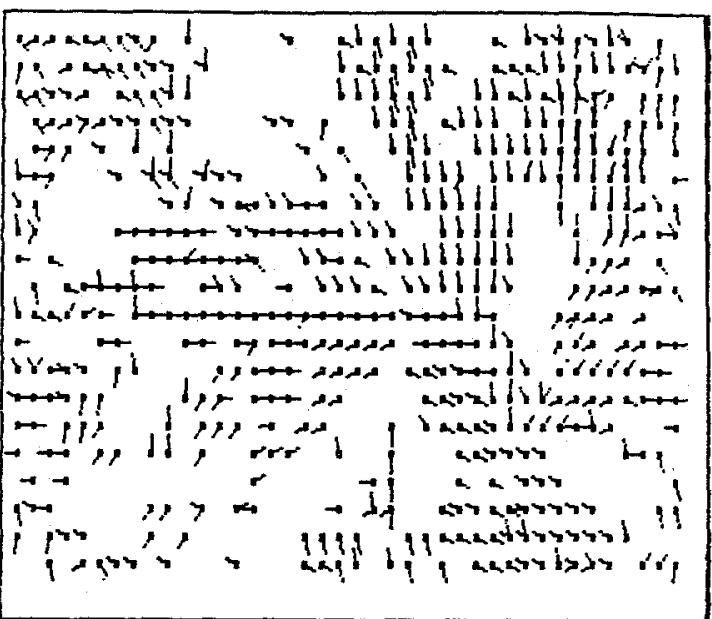

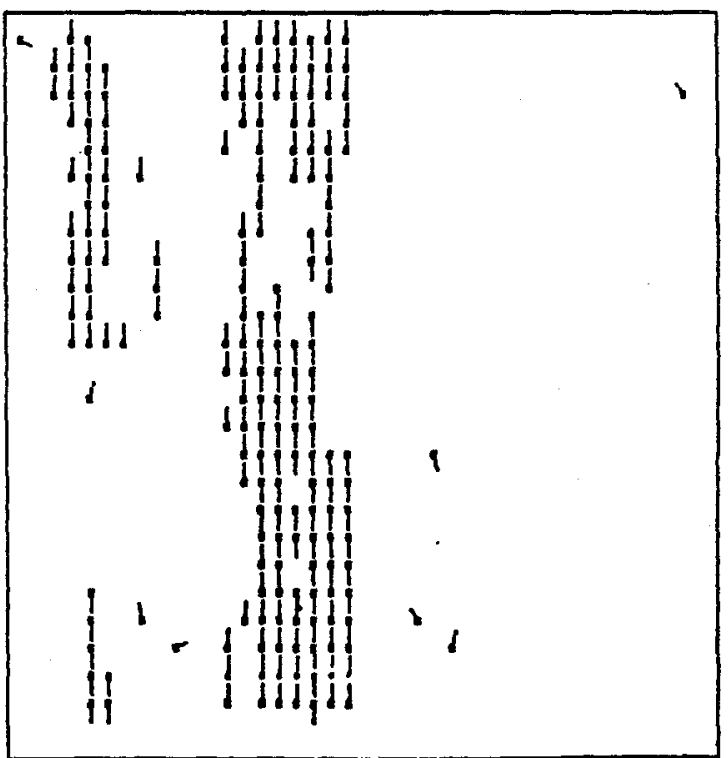

Fig. 7. Cellular automata simulations of interacting objects reveal some of the bifurcation phenomena described in this paper. (a) Formation of several distinct patches of similarly aligned objects. (Note that each patch has its own orientation). (b) Formation of a single alignment region. In this case, the patch is elongated in the direction of its alignment. See EKE (90) and Ermentrout and EK (1991) for details about the cellular automata.

patterns requires scrupulous nonlinear analysis of the competing unstable modes, and will be undertaken later.

\section{Comparison with cellular automata models}

Cellular automata modelling of a similar system was described in EKE (90), Ermentrout and
Edelstein-Keshet (1991). In these models, persistent motion of the objects at random directions, rather than spatial Brownian motion was considered. Furthermore, hard-core repulsion between the objects was introduced. Despite these differences, the qualitative picture of the patterns evolved (see Figs. $7 \mathrm{a}, \mathrm{b}$ ) bear similarities with the qualitative predictions we make in this paper. 
The range of interaction is virtually greatest in the aligned state, so that scenario $B$ is not realized. In some cases, it was clearly seen that patches start to form in the aligned phase, resembling scenario $\mathrm{A}$. The form of the patches is elongated in the same direction as the axis of orientation of the objects within the patches. In other cases (when the density of the objects was higher), patches are formed from aligned objects, but different patches have different orientations. This suggests that scenario $C$ can be realized. We emphasize that cellular automata modelling produces pictures of well-developed patterns, while our paper here deals with the initial phase of this process. No direct correspondence can be made for this reason.

\section{Comparison with physical and biological theories}

There is a close analogy between these models and alignment phenomena described in the physics literature: that of liquid crystals. A good general review of the properties of liquid crystals is found in the book by De Gennes (1974). Liquid crystals are formed by rod-like molecules which undergo random motion and tumbling, and interact by electrostatic attraction or repulsion. Such molecules tend to align with one another, to attract each other at intermediate distance, and repulse at short distances.

In biological examples, (e.g. mammalian cells such as fibroblasts) objects are living units, with essentially "infinite resources of energy" on which to draw. Their interactions cannot be easily summarized with simple physics. The alignment of populations of cells is not an outcome of the shapes of the cells, but of the complex membrane and cellular cytoskeleton, and the dynamic response to contact with another cell.

In work dating back to Onsager (1949) and Zwanzig (1963) the case of hard rod-like molecules which do not overlap was studied using thermodynamic principles. It was shown that thermodynamic considerations of entropy alone, without forces of attraction between molecules could account for the long-range orientational order in these liquid crystals.

Several rigorous models in the physics literature predict orientational phase transitions of the second order for nematics, and these are analogous to the ones we observed in our models. A key difference in the methods of approach and the tools used for analysis of these physical phenomena must be emphasized. Models in physics are traditionally based on minimization of a free energy functional, which mandates that the system studied is being investigated close to thermodynamic equilibrium. However, in our approach the interest is on the dynamic process itself, far away from such equilibrium.

The reason that we abandon the traditional physics approach when dealing with these biological systems is that a meaningful definition of free energy cannot be derived from first principles in highly non-equilibrium open systems such as living cells. It would be possible to formally define a free-energy functional, perhaps, as has been done for some open non-equilibrium systems, but this approach may be artificial. This alternate energy approach is explored in Murray (1989, Section 9.6).

It is interesting to note that there are two groups whose work on liquid crystals is vaguely in the flavour of our approach. The first due to Villard Baron (1969) and Chandrasekhar et al. (1970), is a Monte Carlo simulation of the finite system of elongated molecules represented by objects (ellipses) which undergo thermal transitions restricted by excluded volumes (the ellipses are not allowed to overlap). It is found that a sequence of two transitions-positional and orientational - is observed as the density of the objects increases. For less elongated ellipses, when the homogeneous steady state is disturbed, first the positional transition occurs (patches of spatially ordered ellipses formed) and only then the rotational transition. For more elongated 
ellipses, the order of the transitions is reversed. In these simulations, direct interactions between molecules is absent. However, due to the effect of excluded volumes it is most entropically favorable for them to take on some degree of spatio-angular order. There is clear analogy of these results on the sequence of phase transitions with our scenarios $\mathrm{A}$ and $\mathrm{C}$.

An example of an approach that resembles our model in the field of liquid crystals is the paper by Greco and Marrucci (1992). They also postulate a dynamic system of nonlinear PDE's to describe the interactions of rod-like molecules, and their distribution over space and angle. The details of the equations, and the techniques and results of the analysis is, however, quite different from our own.

There are many other applications of integral equations in the biological literature. We draw the reader's attention to applications concerned with biological movement (Alt, 1988, Othmer et al., 1988), and with neuronal interactions Ermentrout and Cowan, 1979; Ermentrout et al. (1986); Swindale, (1980), (1982), (1991), (1992). Integral equations have also appeared recently in the literature on coupled oscillators. An evolution equation for the density of oscillators at phase $\theta$ and frequency $\omega$, analogous to a model by Kuramoto (1975) was derived and analyzed by Strogatz and Mirollo (1991). The oscillators interact with one another with intensity that depends on their relative phases, and this causes changes in the frequency and phase. An equation described by Strogatz (and reviewed also in Strogatz, 1993) is:

$\frac{\partial \rho}{\partial t}+\frac{\partial(\rho v)}{\partial \theta}=0$

where

$$
\begin{aligned}
& v(\theta, t, \omega)=\omega \\
& +K \int_{-\pi}^{\pi} \int_{-\infty}^{\infty} \sin (\phi-\theta) \rho(\phi, t, \nu) g(\nu) \mathrm{d} \nu \mathrm{d} \phi .
\end{aligned}
$$

This equation bears similarity to Model II discussed in this paper. It is likely that methods outlined in this paper may prove suitable to further investigation of such models.

\section{Acknowledgements}

L.E.K. is supported by NSERC grant OGPIN 021. A.M. is supported by a UGF fellowships from the University of British Columbia. A NATO collaborative research grant is currently funding scientific exchange with $\mathrm{W}$. Alt (Bonn, Germany) on topics related to this research. We would like to thank G.B. Ermentrout, W. Alt, and E. Geigant for discussions about this research.

\section{Appendix}

We consider Models I-III. Results are given below and summarized in Table 1.

\section{Model I}

We consider separately three cases, I: $n=0$, II: $n \neq 0, \hat{K}_{n}>1 / 2$, III: $n \neq 0, \hat{K}_{n}<1 / 2$. We will denote as $g_{n}(q)$ the right hand side of the inequality (4.3).

Case 1 . The function

$$
g_{0}(q)=\mathrm{e}^{-q^{2 / 2}}\left(1-\mathrm{e}^{-q^{2 / 2}}\right)
$$

has the asymptotes: 
Table 1

Summary of the results of linear stability analysis

\begin{tabular}{|c|c|c|c|c|}
\hline & Case I & Case II & & Case III \\
\hline Angular mode & $n=0$ & $n \neq 0$ & & $n \neq 0$ \\
\hline Model & & $\begin{array}{l}\text { I: } \hat{K}_{n}>1 / 2 \\
\text { II: } \hat{V}_{n}>n^{2} \hat{W}_{n} / 2 \\
\text { III: } \hat{G}_{n}>0\end{array}$ & & $\begin{array}{l}\text { I: } \hat{K}_{n}<1 / 2 \\
\text { II: } \hat{V}_{n}<\text { RHS } \\
\text { III: } \hat{G}_{n}<0\end{array}$ \\
\hline Critical wavenumber & $q^{(c)} \simeq 0$ & $q^{(c)}=0$ & $q^{(c)} \neq 0$ & $q^{(c)}=0$ \\
\hline$\epsilon_{1}$ & any & $\epsilon_{1}<\epsilon_{1}^{(c)}$ & $\epsilon_{1}^{(c)}<\epsilon_{1}<\epsilon_{1}^{\prime}$ & $\epsilon_{1}<\epsilon_{1}^{(c)}$ \\
\hline$\epsilon_{2}$ & $\epsilon_{2}<\epsilon_{2}^{(c)}$ & $\epsilon_{2}>\epsilon_{2}^{(c)}$ & $\epsilon_{2}<\epsilon_{2}^{(c)}$ & any \\
\hline Scenario & B & $\mathrm{A}$ & $\mathrm{C}$ & A \\
\hline Figure & $5 b$ & $5 a$ & $4 c$ & $4 a$ \\
\hline Growth rate diagram (Fig.) & 8 & $8 b$ & $8 \mathrm{~b}$ & $8 c$ \\
\hline Linear growth rate (Fig.) & 9 & $9 a$ & $9 b$ & $9 \mathrm{c}$ \\
\hline Stability diagram (Fig.) & $10 a$ & $10 \mathrm{~b}$ & $10 \mathrm{~b}$ & $10 \mathrm{c}$ \\
\hline
\end{tabular}

$$
g_{0}(q) \approx \begin{cases}\frac{1}{2} q^{2}-\frac{3}{8} q^{4}+O\left(q^{6}\right), & q \ll 1, \\ \mathrm{e}^{-q^{2 / 2}}, & q \gg 1,\end{cases}
$$

and the maximum value, $g_{0}(q)=1 / 4$ at $q=q_{0}=(2 \ln (2))^{1 / 2}$. If $\epsilon_{2}>\epsilon_{2}^{(c)}=1 / 2$ then the LGR $\lambda_{0}(q)<0$. If $\epsilon_{2}<\epsilon_{2}^{(c)}$ then $\lambda_{0}(q)<0$ whenever $q>q^{(c)}$ and $\lambda_{0}(q)>0$ whenever $0<q<q^{(c)}$ where

$$
q^{(c)} \approx\left(\frac{4-8 \epsilon_{2}}{3}\right)^{1 / 2}
$$

We show the left and right sides of the inequality (4.3) on the LGR diagram (see Fig. 8a). The LGR is maximal

$$
\lambda_{\max } \approx\left(\frac{1-2 \epsilon_{2}}{3}\right)^{2}
$$

at $q=q_{\max } \approx q^{(c)} / 2$ (see Fig. 9a). Finally, we sketch the stability diagram in the governing parameter space (Fig. 10a). Here the shaded region is the region of stability, (the other region corresponds to instability to the mode $n=0$.)

Case II. In this case, the LGR diagram is as shown in Fig. $8 \mathrm{~b}$. The function $g_{n}(q)$ has the asymptotes

$$
\begin{aligned}
& g_{n}(q) \approx \begin{cases}g_{n}(0)+\hat{K}_{n}\left(2 \hat{K}_{n}-1\right) \frac{q^{2}}{2}-\frac{\hat{K}_{n}\left(4 \hat{K}_{n}-1\right) q^{4}}{8}+O\left(q^{6}\right), & q \ll 1, \\
-\epsilon_{1} n^{2}+\hat{K}_{n} \mathrm{e}^{-q^{2}}, & q \gg 1,\end{cases} \\
& g_{n}(0)=-\epsilon_{1} n^{2}+\hat{K}_{n}\left(1-\hat{K}_{n}\right)
\end{aligned}
$$

and $\max g_{n}(q)=g_{n}(0)+1 / 4$ at

$$
q_{0}=\left(2 \ln \left(2 \hat{K}_{n}\right)\right)^{1 / 2} \text {. }
$$

If $\epsilon_{1}<\epsilon_{1}^{(c)}$ where $\epsilon_{1}^{(c)}$ is defined as

$$
-\epsilon_{1}^{(c)} n^{2}+\hat{K}_{n}\left(1-\hat{K}_{n}\right)=0,
$$

then $\lambda_{n}(q)<0$ if $q>q^{(c)}$ where 
(a)

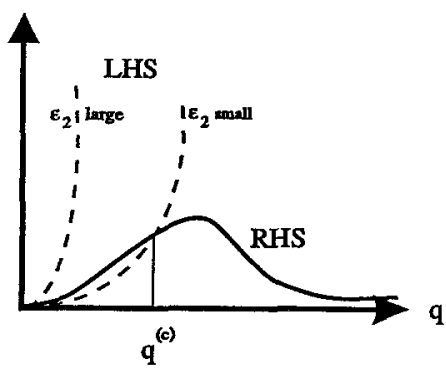

(b)

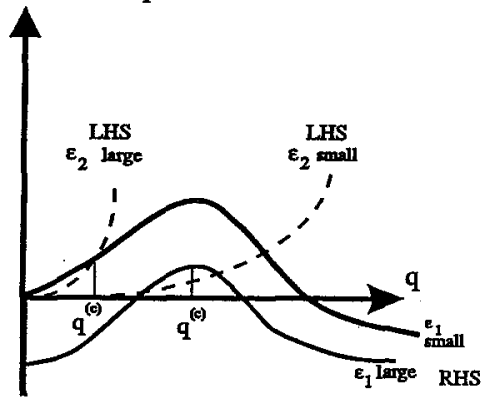

(c)

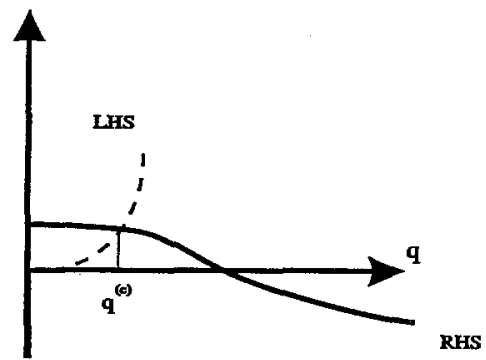

Fig. 8. The linear growth rate diagrams. The dashed lines show the left-hand sides of the instability criteria of the three models. Solid lines are the right hand sides of these criteria. Instability occurs whenever the dashed line is below the solid line. (a) Case I: the unstable mode is $n=0, q=q^{(c)} \approx 0$. (b) Case II: depending on the values of $\epsilon_{1}, \epsilon_{2}$, two possibilities occur. If $\epsilon_{2}$ is large, and $\epsilon_{1}$ is small, we have a situation similar to (a) above. If $\epsilon_{2}$ is smail and $\epsilon_{1}$ is large, the mode $n \neq 0, q=q^{(c)} \neq 0$ is the leading one. (c) Case III: the mode $n \neq 0, q=0$ is unstable. (a)

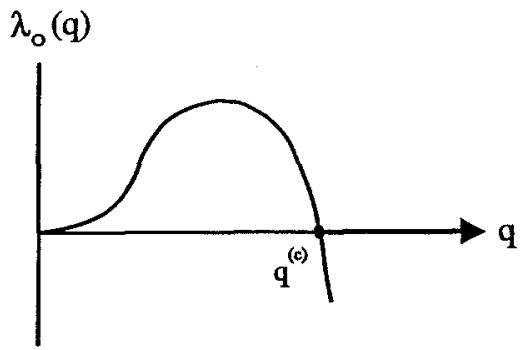

(b)

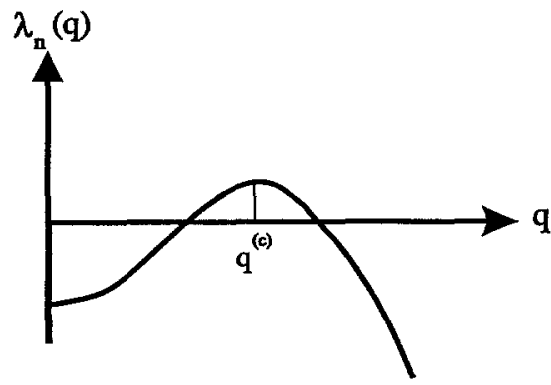

(c)

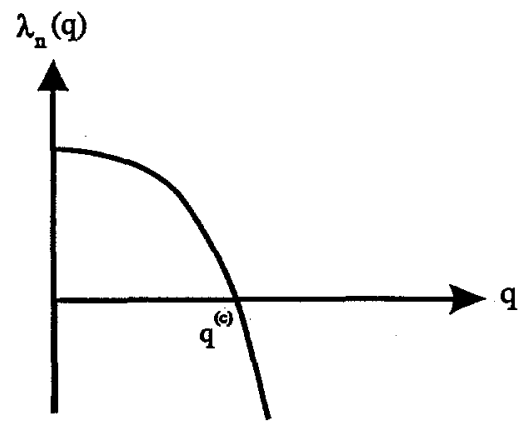

Fig. 9. The linear growth rate of an unstable mode as a function of $q$. (a) and (c) correspond to Figs. 8a,c respectively. (b) corresponds to Fig. $8 \mathrm{a}$ if $\epsilon_{2}$ is large, and to Fig. $8 \mathrm{~b}$ if $\epsilon_{2}$ is small.

$$
q^{(c)} \approx n\left(\frac{\epsilon_{1}-\epsilon_{1}^{(c)}}{\epsilon_{2}}\right)^{1 / 2}
$$

at any $\epsilon_{2}$. Otherwise, $\lambda_{n}(q)>0$. If $\epsilon_{1}>\epsilon_{1}^{(c)}$ then $\lambda_{n}(q)<0$ at any $q$, whenever $\epsilon_{2}>\epsilon_{2}^{\prime}\left(\epsilon_{1}\right)$ and $\lambda_{n}(q)>0$ at $q \approx q_{0} \neq 0$ whenever $\epsilon_{2}<\epsilon_{2}^{\prime}\left(\epsilon_{1}\right)$, where

$$
\epsilon_{2}^{\prime}\left(\epsilon_{1}\right) \approx \frac{1-4 \epsilon_{1} n^{2}}{4 q_{0}^{2}}, \quad \epsilon_{2}^{\prime}\left(\epsilon_{1}\right)<\frac{1}{2} \hat{K}_{n}\left(2 \hat{K}_{n}-1\right)<\frac{1}{2}, \quad \epsilon_{1}<\frac{1}{4 n^{2}} .
$$

The LGR is maximal at $q=0$ at $\epsilon_{1}<\epsilon_{1}^{(c)}$ (see Fig. 9a). It is maximal if $q \approx q_{0} \neq 0$ if 
(a)

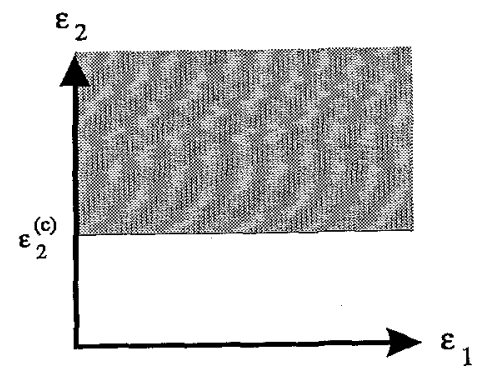

(b)

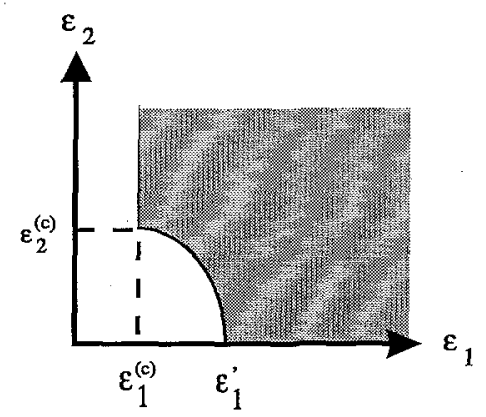

(c)

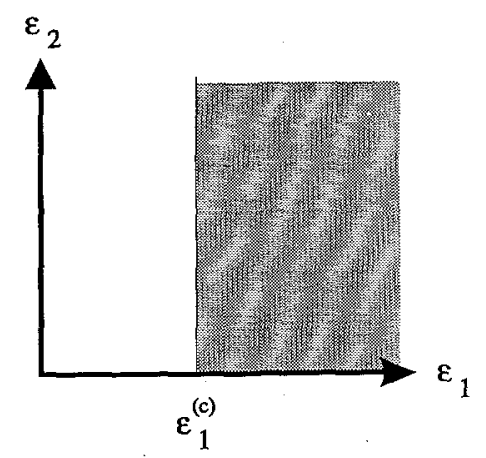

Fig. 10. The stability diagram in the governing parameter space. The shaded region is the stability region and the unshaded region corresponds to a non-homogeneous pattern. (a) Case I: Independently of $\epsilon_{1}$, when $\epsilon_{2}$ decreases below $\epsilon_{2}^{(c)}$, the orientationally disordered patches start to grow (scenario B). (b) Case II: the vertical border of the shaded region corresponds to the growing mode $n \neq 0, q=0$ (scenario A). The curved border corresponds to the growing mode $n \neq 0, q \neq 0$ (scenario C). (c) Case III: Independently of $\epsilon_{2}$, as $\epsilon_{1}$ decreases below $\epsilon_{1}^{(c)}$, the mode $n \neq 0, q=0$ breaks the stability (scenario A).

$$
\epsilon_{1}^{(c)}<\epsilon_{1}<\frac{1}{4 n^{2}}, \quad \epsilon_{2}<\epsilon_{2}^{\prime}\left(\epsilon_{1}\right)
$$

(See Fig. 9b). The stability diagram then has the form shown in Fig. 10b.

Case III. In this case, the LGR diagram looks like Fig. 8c. $\lambda_{n}(q)>0$ at $q<q^{(c)}$ whenever $\epsilon_{1}<\epsilon_{1}^{(c)}$ and any $\epsilon_{2} . \lambda_{n}(q)$ is the same as the one shown in Fig. 9c. The stability diagram is shown in Fig. 10c. 
To determine the primary bifurcation in the case that $\epsilon_{1}$ and $\epsilon_{2}$ decrease monotonously, we must find the intersection of a countable number of subsets of $\mathbb{R}^{2}$ corresponding to the shaded stability regions discussed above (for all $n=0,1,2, \ldots$ ). Then the border of this intersection is the line of the primary bifurcation. The borders of the stability areas which do not belong to this line together form lines of secondary bifurcations. The cumulative set of bifurcation lines is shown in Fig. 4. We call this picture the Complete Bifurcation Diagram.

\section{Model II}

It is easy to see that the right hand side of the inequality (4.5) has the following asymptotes:

$$
g_{n} \approx \begin{cases}\left(-\epsilon_{1} n^{2}+\bar{C} n^{2} \hat{W}_{n}\right)+\bar{C}\left(\hat{V}_{n}-\frac{n^{2}}{2} \hat{W}_{n}\right) q^{2}+O\left(q^{4}\right), & q \ll 1, \\ -\epsilon_{1} n^{2}+\bar{C} \hat{V}_{n} q^{2} \mathrm{e}^{-q^{2} / 2}, & q \gg 1 .\end{cases}
$$

Furthermore, the function $g_{n}(q)$ has a maximum at $q=0$ if $\hat{V}_{n}<n^{2} \hat{W}_{n} / 2$ and at $q \neq 0$ if $\hat{V}_{n}>n^{2} \hat{W}_{n} / 2$.

We consider separately three cases: I, $n=0 ;$ II, $n \neq 0, \hat{V}_{n}>n^{2} \hat{W}_{n} / 2 ;$ III, $n \neq 0, \hat{V}_{n}<n^{2} \hat{W}_{n} / 2$;

Case $I$. When $n=0$ the LGR diagram is as shown in Fig. 8a. Independently of $\epsilon_{1}$ there is stability at all $q$ 's if $e_{2}>\bar{C} \hat{V}_{n}$. There is instability at small $q$ 's if $\epsilon_{2}<\bar{C} \hat{V}_{n}$. The linear growth rate and the stability diagram are shown in Figs. 9a and 10a respectively.

Case II. When $n \neq 0, \hat{V}_{n}>n^{2} \hat{W}_{n} / 2$, the LGR diagram is given in Fig. 8b. If $\epsilon_{2}>\epsilon_{2}^{(c)}$ and $\epsilon_{1}>\epsilon_{1}^{(c)}$, where

$$
\epsilon_{2}^{(c)}=\bar{C}\left(\hat{V}_{n}-\frac{n^{2} \hat{W}_{n}}{2}\right), \quad \epsilon_{1}^{(c)}=\bar{C} \hat{W}_{n}
$$

there is stability at all $q$ 's. If $\epsilon_{2}>\epsilon_{2}^{(c)}, \epsilon_{1}<\epsilon_{1}^{(c)}$ there is instability at small $q$ 's. At $\epsilon_{2}<\epsilon_{2}^{(c)}, \epsilon_{1}^{(c)}<\epsilon_{1}<\epsilon_{1}^{\prime}$ there is stability at all $q$ 's if $\epsilon_{2}>\epsilon_{2}^{\prime}\left(\epsilon_{1}\right)$ and instability at $q \approx q^{(c)} \neq 0$ if $\epsilon_{2}<\epsilon_{2}^{\prime}\left(\epsilon_{1}\right)$. Here $\epsilon_{1}^{\prime}$ and $\epsilon_{2}^{\prime}\left(\epsilon_{1}\right)$ depend parametrically on $\hat{V}_{n}, \hat{W}_{n}$, and $\bar{C}$ and can be found from certain transcendental equations. $q^{(c)}$ is defined by the expression:

$$
q^{(c)} \approx\left(2-\frac{\hat{W}_{n} n^{2}}{\hat{V}_{n}}\right)^{1 / 2}
$$

The linear growth rate and the stability diagram are given in Figs. 9a, 9b, and 10b.

Case III. The LGR diagram in the case $n \neq 0, \hat{V}_{n}<n^{2} \hat{W}_{n} / 2$ is given in Fig. 8c. Independently of $\epsilon_{2}$, there is stability at all $q$ 's, if $\epsilon_{1}>\epsilon_{1}^{(c)}$ and there is instability at small $q$ 's if $\epsilon_{1}<\epsilon_{1}^{(c)}$ where

$$
-\epsilon_{1}^{(c)}+\bar{C} \hat{W}_{n}=0
$$

(if $\hat{W}_{n}<0$ there is no instability at all.) The linear growth rate and the stability diagram are shown in Figs. 9c, 10c respectively.

Because $\epsilon_{2}^{(c)}<\bar{C} \hat{V}_{n}$, the complete bifurcation diagram has the same form as the one in Fig. 4 .

\section{Model III}

The right hand side of the inequality (4.7) has the asymptotes:

$$
g_{n}(q) \approx \begin{cases}\left(-\epsilon_{1} n^{2}+\eta \bar{C}^{2}\left(1-\hat{G}_{n}\right)\right)+\frac{\eta}{2} \bar{C}^{2} \hat{G}_{n} q^{2}+O\left(q^{4}\right), & q \ll 1, \\ \left(-e_{1} n^{2}+\eta \bar{C}^{2}\right)-\eta \bar{C}^{2} \hat{G}_{n} \mathrm{e}^{-q^{2 / 2}}, & q \gg 1,\end{cases}
$$


$g_{n}(q)$ is monotonously decreasing if $\hat{G}_{n}<0$ and monotonously increasing if $\hat{G}_{n}>0$. Here we have to distinguish three cases: I, $n=0 ; \mathrm{II}, n \neq 0, \hat{G}_{n}>0$; III, $n \neq 0, \hat{G}_{n}<0$.

Case I. The LGR diagram for the case $n=0$ is shown in Fig. 8a. Independently of $\epsilon_{1}$ there is stability at all $q$ 's if $\epsilon_{2}>\eta \bar{C} / 2$ and there is instability at small $q$ 's if $\epsilon_{2}<\eta \bar{C}^{2} / 2$. The linear growth rate and stability diagram are given in the Figs. 9a, 10a respectively.

Case II. If $n \neq 0, \hat{G}_{n}>0$, the LGR diagram is similar to that shown in Fig. $8 \mathrm{~b}$. The only difference is that the humped curves are monotonically increasing in this case, a feature which does not alter the results. If $\epsilon_{2}>\epsilon_{2}^{(c)}$ and $\epsilon_{1}>\epsilon_{1}^{(c)}$, where

$$
\epsilon_{2}^{(c)}=\frac{\eta \bar{C}^{2} \hat{G}_{n}}{2}, \quad \epsilon_{1}(c)=\eta \bar{C}^{2} \frac{\left(1-\hat{G}_{n}\right)}{n^{2}},
$$

there is stability at all $q$ 's. At $\epsilon_{2}>\epsilon_{2}^{(c)}$ and $\epsilon_{1}<\epsilon_{1}^{(c)}$, there is instability at small $q$ 's. At $\epsilon_{2}<\epsilon_{2}^{(c)}$, $\epsilon_{1}^{(c)}<\epsilon_{1}\left\langle\eta \bar{C}^{2} / n^{2}\right.$ there is stability at all $q$ 's. If $\epsilon_{2}>\epsilon_{2}^{\prime}\left(\epsilon_{1}\right)$ there is instability at some $q \approx q^{(c)} \neq 0$ if $\epsilon_{2}<\epsilon_{2}^{\prime}\left(\epsilon_{1}\right)$. Here $\epsilon_{2}^{\prime}\left(\epsilon_{1}\right)$ depends parametrically on $\eta, \bar{C}, \hat{G}_{n}$, and can be found from certain transcendental equations. The distinctive feature of this model is that $q^{(c)}$ can vary from zero to infinity:

$$
q^{(c)} \rightarrow \infty \quad \text { if } \epsilon_{2} \rightarrow 0, \epsilon_{1} \rightarrow \frac{\eta \bar{C}^{2}}{n^{2}} .
$$

The linear growth rate and stability diagram are shown in Figs. 9a, 9b, 10b.

Case III. If $n \neq 0, \hat{G}_{n}<0$, the LGR diagram is shown in Fig. 8c. Independently of $\epsilon_{2}$, there is stability at all $q$ 's if $\epsilon_{1}>\epsilon_{1}^{(c)}$ and instability at small $q$ 's if $\epsilon_{1}<\epsilon_{1}^{(c)}$ where $\epsilon_{1}^{(c)}$ is given in (A.16). The linear growth rate and stability diagram are shown in Figs. $9 \mathrm{c}$ and 10c, respectively. Because

$$
\epsilon_{2}^{(c)}=\frac{\eta \bar{C}^{2}}{2} \hat{G}_{n}<\frac{\eta \bar{C}^{2}}{2}
$$

the complete bifurcation diagram has the same form as the one in Fig. 4.

\section{References}

Alt, W. and Geigant, E. (1994), private communication.

Abramowitz, M. and Stegun, A. eds. (1964), Handbook of Mathematical Functions (National Bureau of Standards, Washington). Alt, W. (1988), Modelling of motility in biological systems, ICIAM 1987 Proceedings (SIAM, Philadelphia) pp. 15-30.

Civelekoglu, G. and Edelstein-Keshet, L. (1994), Models for the formation of actin structures, Bull. Math. Biol. 56, 587-616.

Chandrasekhar, S., Shashihad, R. and Tara, N. (1970), Theory of melting of molecular crystals: the liquid crystalline phase, Mol. Cryst. Liq. Cryst. 10, 337-358.

De Gennes, P.G. (1974), The Physics of Liquid Crystals (Clarendon, Oxford).

Edelstein-Keshet, L. and Ermentrout, G.B. (1989), Models for branching networks in two dimensions, SIAM J. Appl. Math. 49, 1136-1157.

Edelstein-Keshet, L. and Ermentrout, G.B. (1990), Models for contact-mediated pattern formation: cells that form parallel arrays, J. Math. Biol. 29, 33-58.

Edmunds, M. (1974), Defense in Animals (Longman, London).

Elsdale, T. (1972), Pattern formation in fibroblast cultures, an inherently precise morphogenetic process, in: Towards a Theoretical Biology, Vol. 4, C.H. Waddington, ed. (Edinburgh Univ. Press, Edinburgh).

Elsdale, T. and Wasoff, F. (1976), Fibroblast cultures and dermatoglyphics: the topology of two planar patterns: Wilhelm Roux's Archives, Vol. 180, pp. 121-147.

Ermentrout, B., Campbell, J. and Oster, G. (1986), A model for shell patterns based on neural activity, The Veliger 28, 369-388. Ermentrout, G.B. and Cowan, J. (1979), A mathematical theory of visual hallucination patterns, Biol. Cybern. 34, $137-150$. 
Ermentrout, G.B. and Edelstein-Keshet, L. (1991), Cellular Automata approaches to biological modelling, J. Theor. Biol. 160, 97-133.

Geigant, E., Mogilner, A. and Ladizhansky, K. (1995), A nonlinear model for active alignment, in preparation.

Greco, F. and Marrucci, G. (1992), Rodlike molecular dynamics, the tumbling regime, Mol. Cryst. Liq. Cryst. 212 , $125-137$.

Gross, M.C. and Hohenberg, P.C. (1993), Pattern formation outside of equilibrium, Rev. Mod. Phys. 65, 851-112.

Grunbaum, D. (1994), Swarming behaviour as an aide to Chemotaxis; in: Parrish, J. and Hammner, W., eds., 3D Animal Aggregations (Cambridge Univ. Press, Cambridge).

Katz, L.C., Potel, M.J. and Wasserung, R.J. (1981), Structure and mechanism of schooling in tadpoles of the clawed frog, Xenopus laevis, Anim. Behav. 29, 20-33.

Kuramoto, Y. (1975), Self-entrainment of a population of coupled nonlinear oscillators, in: Intern. Symp. on Mathematical Problems in Theoretical Physics, H. Araki, ed., Lecture Notes in Physics, Vol. 39 (Springer, New York) pp. $420-422$.

Landau, L.D. and Lifshitz, E.M. (1956), Hydrodynamics (Pergamon, London).

Lifshitz, E.M. and Pitaevskii, L.P. (1981), Physical Kinetics (Pergamon, Oxford).

Macrobert, T.M. (1967), Spherical Harmonics (Pergamon, Oxford).

Marcus, M. and Ming, H. (1964), A Survey of Matrix Theory and Matrix Inequalities (Allyn Bacon, Boston).

Mogilner, A. and Edelstein-Keshet, L. (1995a), Selecting a common direction I: How orientational order can arise from simple contact responses between interacting cells, J. Math. Biol., in press.

Mogilner, A. and Edelstein-Keshet, L. (1995b), Selecting a common direction II: Peak-like solutions representing total alignment of cell clusters, submitted for publication.

Murray, J.D. (1989), Mathematical Biology (Springer, New York).

O'Brien, D.P. (1989), Analysis of the internal arrangement of individuals within crustacean aggregations (Euphausiacea, Mysidacea), J. Exp. Mar. Biol. Ecol. 128, 1-30.

Onsager, L. (1949), The effects of shape on the interaction of colloidal particles, Ann. NY Acad. Sci. 51, 627-659.

Othmer, H.G., Dunbar, S.R. and Alt, W. (1988), Models of dispersal in biological systems, J. Math. Biol. 26, $263-298$.

Pfistner, B. and Alt, W. (1989), A two-dimensional random walk model for swarming behaviour, Alt, W. and Hoffmann, G., eds., in: Biological Motion (Springer, New York).

Segel, L. (1984), Modelling Dynamic Phenomena in Molecular and Cellular Biology (Cambridge Univ. Press, Cambridge).

Strogatz, S.H. (1993), Norbert Wiener's brain waves, in: Lecture Notes in Biomathematics, Vol. 100 (Springer, Berlin).

Strogatz, S.H. and Mirollo, R.E. (1991), Stability of incoherence in a population of coupled oscillators, J. Stat. Phys. 63, 613-635.

Swindale, N.V. (1980), A model for the formation of ocular dominance stripes, Proc. R. Soc. Lond. B $208,243-264$.

Swindale, N.V. (1982), A model for the formation of orientation columns, Proc. R. Soc. London B 215, $211-230$.

Swindale, N.V. (1991), Coverage and the design of striate cortex, Biol. Cybern. 65, 415-424.

Swindale, N.V. (1992), A model for the coordinated development of columnar systems in primate striate cortex, Biol. Cybern. 66, 217-230.

Tabony, J. and Job, D. (1990), Spatial structures in microtubular solutions requiring a sustained energy source, Nature 346, 448-451.

Villard Baron, J. (1969), J. Phys. (Paris) 30 (Suppl. 34), 22.

Zwanzig, R. (1963), First-order phase transitions in a gas of long thin rods, J. Chem. Phys. 39, $1714-1721$. 